\title{
Avaliação da Goma Guar no desenvolvimento de comprimidos matriciais de liberação controlada de teofilina
}

\section{Evaluation of Guar Gum in the development of theophylline controlled-release matrix tablets}

\author{
Edilene Gadelha de Oliveira1', Rosana de Sousa Campos², Anaiara Silva Machado³, \\ Juliana Fernandes Pereira ${ }^{4}$ e Tamara Gonçalves de Araúj0 ${ }^{3 *}$ \\ 'Departamento de Farmácia, Centro de Ciências da Saúde, Universidade Federal do \\ Rio Grande do Norte - UFRN, Natal, RN, Brasil \\ 2Programa de Pós-graduação em Microbiologia Médica, Faculdade de Medicina, \\ Universidade Federal do Ceará - UFC, Fortaleza, CE, Brasil \\ ${ }^{3}$ Departamento de Farmácia, Faculdade de Farmácia, Odontologia e Enfermagem, \\ Universidade Federal do Ceará - UFC, Fortaleza, CE, Brasil \\ ${ }^{4}$ Programa de Pós-graduação em Ciências Médicas, Faculdade de Medicina, \\ Universidade Federal do Ceará - UFC, Fortaleza, CE, Brasil \\ *tamara.ufc@gmail.com
}

\begin{abstract}
Resumo
O objetivo desse estudo foi formular e avaliar comprimidos de liberação controlada. Comprimidos de liberação controlada de teofilina foram preparadas pelo método de compressão direta usando dois polímeros como, o HPMC K 100M (polímero hidrofílico) e a Goma Guar (Polímero natural), isolado ou em mistura (GG:HPMC 3:1) e GG:HPMC 1:3). Os comprimidos foram caracterizados através do peso médio, diâmetro, altura, dureza, friabilidade. Todos os resultados estiveram em conformidade com os limites aceitáveis. O ensaio de intumescimento foi realizado em água destilada durante 4 horas, sendo determinado pela diferença de peso do comprimido seco e intumescido. $\mathrm{O}$ ensaio de dissolução foi realizado em água destilada $\left(900 \mathrm{~mL}, 37 \pm 0,5^{\circ} \mathrm{C}, 50 \mathrm{rpm}\right.$, aparato II) durante 8 horas. Os resultados demonstraram que a Goma guar isolada não possui capacidade de retardar a liberação da teofilina por 8 horas. Comprimidos matriciais contendo GG:HPMC (3:1) apresentaram um melhor controle de liberação da teofilina.
\end{abstract}

Palavras-chave: Goma Guar, teofilina, comprimidos, liberação controlada.

\section{Abstract}

Guar gum (GG) has been widely used in drug delivery systems due to its low cost and nontoxicity. Theophylline controlled release tablets were prepared by directly compressed method using two polymers such as HPMC K 100M (hydrophilic polymer) and Guar Gum (natural polymer), isolated or in mixture (GG:HPMC 3:1 and GG:HPMC 1:3). The formulated tablets were evaluated for: weight variation, diameter, height, hardness and friability. All the parameters were within the acceptable limits. The swelling test was performed in distilled water for 4 hours, it was determined by the difference in weight of the dry and swollen tablet. The dissolution test was performed in distilled water $(900 \mathrm{~mL}$, $37 \pm 0.5^{\circ} \mathrm{C}, 50 \mathrm{rpm}$, apparatus II) for 8 hours. The results showed that guar gum alone could not control the theophylline release effectively for 8 hours. Matrix tablet prepared from GG: HPMC (3:1) is a better formulation for the sustained released matrix tablets of theophylline.

Keywords: Guar Gum, theophylline, tablets, controlled release.

\section{Introdução}

A tecnologia farmacêutica associada à modificação da liberação de fármacos, a partir de preparações farmacêuticas, sofreu um incremento notório nas últimas décadas na tentativa de maximizar as vantagens inerentes às formas farmacêuticas de liberação controlada ${ }^{[1]}$. O sucesso dessas novas tecnologias caracteriza-se pela total desvinculação destas propostas com os elevados investimentos previstos à descoberta de novos fármacos ${ }^{[2]}$. 
A principal vantagem dos comprimidos matriciais de liberação controlada consiste na tentativa de manter as concentrações plasmáticas do fármaco a níveis terapêuticos. Estas diminuem as flutuações da dose administrada, eliminando a necessidade de administrações frequentes, melhorando a adesão do paciente ao tratamento ${ }^{[3,4]}$.

Os sistemas matriciais constituídos por polímeros é uma das estratégias mais empregadas no desenvolvimento de uma formulação oral de liberação modificada devido às vantagens inerentes a estes sistemas: versatilidade, eficácia, baixo custo e produção que recorre a equipamentos e técnicas convencionais $^{[5]}$.

No desenvolvimento de sistemas matriciais, deve-se levar em consideração a estrutura da matriz, a cinética de liberação, os mecanismos para controlar a liberação do fármaco (erosão, difusão, intumescimento), a natureza química e as propriedades dos materiais utilizados. $\mathrm{O}$ mecanismo de liberação dos fármacos é dependente de fatores como a solubilidade do fármaco, capacidade de hidratação/ intumescimento do polímero e formação de camada gelatinosa que funciona como uma barreira que controla a velocidade de liberação do fármaco ${ }^{[6]}$.

Quando uma matriz hidrofílica entra em contato com o fluido do trato gastrointestinal, ocorre uma hidratação das moléculas mais superficiais da interface sólido-líquido, seguido por rearranjo da cadeia polimérica, formando uma camada gelatinosa. À medida que a água penetra no sistema, a camada de gel vai aumentando, enquanto que as camadas mais externas podem sofrer um processo de erosão. Em seguida, ocorre uma total geleificação do sistema e liberação do fármaco por difusão, erosão ou ambos ${ }^{[7]}$.

A hidroxipropilmetilcelulose (HPMC) é um polímero hidrossolúvel muito utilizado como agente formador de comprimidos matriciais hidrofílicos ${ }^{[8]}$. Uma das suas características mais importantes é a capacidade de intumescimento/relaxamento, a qual exerce efeito pronunciado na liberação do fármaco. $\mathrm{O}$ grau de hidratação deste polímero depende das suas características físico-químicas, como a massa molecular, o grau de substituição e a viscosidade ${ }^{[9]}$.

A Goma guar é um polímero natural hidrossolúvel com interesse na preparação de comprimidos matriciais devido ao baixo custo e a sua habilidade na formação de uma barreira difusional quando intumescida ${ }^{[10]}$. É um polissacarídeo natural extraído das sementes de Cyamopsis tetragonolobus (Leguminosea), constituído por unidades de $\beta$-1,4-manose glicosídica e uma unidade $\alpha$-1,6-galactose a cada duas unidades de manose, formando ramificações ${ }^{[11]}$.

A combinação criteriosa de polímeros, em determinadas concentrações, pode constituir a matriz de um sistema de liberação de fármacos muito promissor, que fornece um efeito terapêutico prolongado e uma biodisponibilidade mais equilibrada do fármaco ${ }^{[10,11]}$. Essa associação tem sido bastante estudada no intuito de garantir maior eficácia sobre o controle da liberação de fármacos ${ }^{[12-14]}$.

A teofilina, fármaco solúvel em água, foi escolhida como modelo devido a sua eficiência no tratamento da asma e doença pulmonar obstrutiva crônica, sendo necessária a sua monitorização plasmática devido aos efeitos colaterais desagradáveis. Diante disso, podem ser usadas formas farmacêuticas de liberação lenta, as quais minimizam as oscilações plasmáticas, no intuito de diminuir efeitos adversos ${ }^{[15]}$. O objetivo deste trabalho foi avaliar o comportamento da Goma guar isolada em relação à hidroxipropilmetilcelulose, em diferentes razões GG:HPMC, no intuito de utilizar esse polímero natural em comprimidos matriciais de liberação controlada da teofilina.

\section{Experimental}

\subsection{Materiais}

Goma guar(All Chemistry, Brasil), Hidroxipropilmetilcelulose (METHOCEL $\left.^{\mathrm{TM}}, \mathrm{K} 4 \mathrm{M}\right)$ fornecida gentilmente pela Colorcon ${ }^{\circledR}$ (Brasil), teofilina anidra (Deg, Brasil), celulose microcristalina (All Chemistry, Brasil), estearato de magnésio (Deg, Brasil), dióxido de silício coloidal (Deg, Brasil).

\subsection{Produção e caracterização dos comprimidos matriciais}

Os componentes da formulação foram pesados em balança analítica (Gehaka, modelo AG 200) e submetidos à uniformidade de granulometría, utilizando um tamiz de malha $355 \mu \mathrm{m}$ (Granutest). Em seguida, a teofilina, a Goma guar, a HPMC e a celulose microcristalina foram misturadas em gral e pistilo. Posteriormente, o estearato de magnésio e o dióxido de silício coloidal foram adicionados à mistura para a preparação dos comprimidos de teofilina $(100 \mathrm{mg})$, utilizando uma máquina de comprimir rotatória semi-automática (Lawes, modelo W216), com punção de $11 \mathrm{~mm}$. Foram produzidos os lotes A (GG), lote B (GG:HPMC 3:1), lote C (GG:HPMC 1:3) e lote D (HPMC). A composição de cada lote pode ser observada na Tabela 1.

\subsection{Determinação do peso médio}

Para a determinação do peso médio, foram individualmente pesados 20 comprimidos. A faixa de tolerância preconizada é de $5 \%$, quando o peso total for superior a $250 \mathrm{mg}^{[16]}$.

Tabela 1. Composição qualitativa e quantitativa dos diferentes lotes dos comprimidos matriciais de teofilina.

\begin{tabular}{lrrrr}
\hline \multicolumn{1}{c}{ Composição $(\mathbf{m g})$} & Lote A & Lote B & Lote C & Lote D \\
\hline Teofilina & 100,00 & 100,00 & 100,00 & 100,00 \\
HPMC & - & 36,30 & 108,70 & 146,00 \\
Goma guar & 146,00 & 108,70 & 36,30 & - \\
Celulose microcristalina & 146,00 & 146,00 & 146,00 & 146,00 \\
Estearato de magnésio & 4,00 & 4,00 & 4,00 & 4,00 \\
Dióxido silício coloidal & 4,00 & 4,00 & 4,00 & 4,00 \\
Total & 400,00 & 400,00 & 400,00 & 400,00 \\
\hline
\end{tabular}




\subsection{Determinação da altura, diâmetro e dureza}

Foram retiradas amostras para determinação da altura e diâmetro $(\mathrm{n}=20)$, utilizando um paquímetro digital (Starrett, modelo 727). A dureza $(n=3)$ foi ajustada durante a produção dos comprimidos e medida através de um durômetro (Nova ética, modelo 298), considerando o intervalo de 70-90 N, acima do valor mínimo permitido de $30 \mathrm{~N}$ pela Farmacopeia Brasileira (2010).

\subsection{Determinação da friabilidade}

A friabilidade dos comprimidos $(n=6)$ foi determinada quando estes foram colocados em um friabilômetro (Logan Instruments, modelo FAB-2), durante um período de 4 minutos, e submetidos a uma velocidade de $25 \mathrm{rpm}$. $\mathrm{O}$ resultado foi obtido pela diferença entre o peso inicial e final, medido em função da porcentagem de massa perdida. O valor preconizado é inferior a $1,5 \%$ do peso estabelecido ${ }^{[16]}$.

\subsection{Grau de intumescimento (Gl)}

Os comprimidos matriciais foram colocados em cestas de dissolutor, imersas em béquer contendo $150 \mathrm{~mL}$ de água destilada, durante 4 horas. Em tempos previamente determinados $(0,25 ; 0,5 ; 1 ; 2 ; 3$ e 4 horas $)$, os comprimidos foram retirados do meio e avaliado o grau de intumescimento $(G I)$ da matriz polimérica, utilizando o método gravimétrico, pela diferença de peso do comprimido seco $(P s)$ e intumescido $(P i)$, através da seguinte relação: $G I(\%)=(P i-P s) / P s \times 100$. O ensaio foi realizado em triplicata ${ }^{[17]}$.

\subsection{Perfil de dissolução in vitro}

O ensaio de dissolução foi realizado em água destilada a $37 \pm 0,5^{\circ} \mathrm{C}$, sob condições sink, utilizando dissolutor com aparato II (Erweka, modelo DT 800), a uma velocidade de agitação de $50 \mathrm{rpm}$ e o volume do meio de dissolução de $900 \mathrm{~mL}$ durante 8 horas de ensaio. A metodologia foi adaptada do método utilizado para cápsulas de liberação prolongada de teofilina ${ }^{[18]}$. Foram retiradas alíquotas de $10 \mathrm{~mL}$ do meio de dissolução em tempos previamente determinados $(0,5 ; 1 ; 2 ; 3 ; 4 ; 6$ e 8 horas). Em seguida, as amostras foram diluídas em água destilada e as leituras foram realizadas em espectrofotômetro (Genesys, modelo $10 \mathrm{UV}$ ) em $272 \mathrm{~nm}$. O ensaio foi realizado em sextuplicata.

\section{Resultados e Discussão}

\subsection{Características físico-químicas dos comprimidos matriciais}

As características físico-químicas dos comprimidos matriciais de teofilina estão apresentadas na Tabela 2. De acordo com os resultados obtidos, observou-se que os comprimidos foram produzidos em conformidade com os padrões farmacopéicos.

\subsection{Grau de intumescimento (GI) e perfil de liberação do fármaco}

De acordo com a Figura 1, observou-se que o lote A(GG) apresentou elevado grau de intumescimento nos primeiros 30 minutos de ensaio, seguido pela perda gradual de água e alta taxa de erosão da matriz. A natureza do polímero é determinante no processo de intumescimento em comprimidos matriciais ${ }^{[19,20]}$. Quando se aumenta a concentração de polissacarídeo na formulação favorece a hidratação do sistema matricial, e consequentemente, a formação de uma camada viscosa gelatinosa, o que influencia diretamente no controle de liberação do fármaco ${ }^{[20]}$.

$\mathrm{Na}$ Figura 2, estão representados os perfis de liberação das formulações contendo GG e/ou HPMC. A matriz polimérica de Goma guar (Lote A) sofreu alto grau de erosão, quando comparado ao intumescimento, perdendo, assim, a capacidade de retenção da liberação do fármaco. Srivastava et al. ${ }^{[10]}$ avaliaram a goma guar e acácia como agentes modificadores de liberação do diclofenaco. Houve uma diminuição da taxa de liberação do fármaco com o aumento do índice de intumescimento do polímero. Isso pode ser atribuído à lenta erosão da camada gelatinosa nos comprimidos matriciais. Contudo, os autores não observaram a influência da GG sozinha na liberação do fármaco e, por isso, sugere-se que o uso de outro polímero foi determinante na diminuição da erosão do sistema. Diante disso, no presente estudo foi utilizada a HPMC em associação à GG em diferentes razões. Após adição de HPMC na matriz, os demais lotes

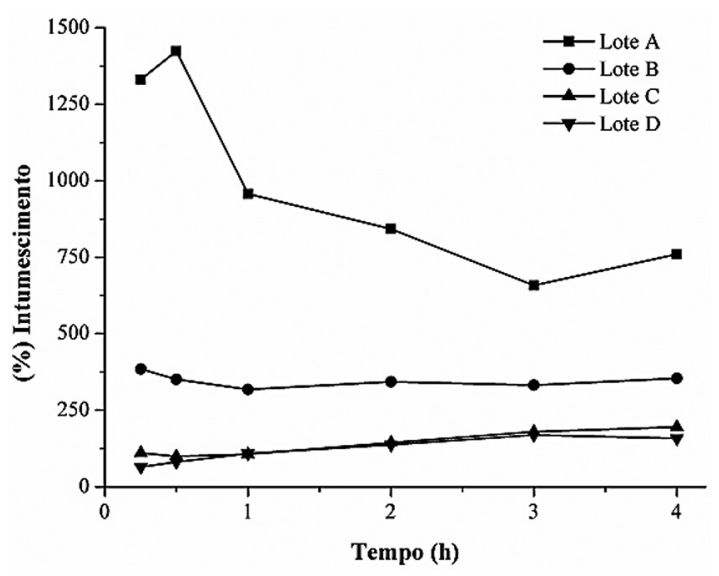

Figura 1. Gráfico do grau de intumescimento (\%) dos lotes de comprimidos matriciais de teofilina durante 4 horas de ensaio.

Tabela 2. Características físico-químicas dos comprimidos matriciais de teofilina.

\begin{tabular}{|c|c|c|c|c|}
\hline Propriedades & Lote A & Lote B & Lote C & Lote D \\
\hline Peso médio $(\mathrm{mg}) \pm \mathrm{CV}$ & $400,70 \pm 0,55$ & $401,70 \pm 0,26$ & $401,20 \pm 0,37$ & $401,55 \pm 0,92$ \\
\hline Diâmetro $(\mathbf{m m}) \pm \mathrm{CV}$ & $11,01 \pm 0,04$ & $10,98 \pm 0,05$ & $11,05 \pm 0,04$ & $11,02 \pm 0,08$ \\
\hline Altura $(\mathrm{mm}) \pm \mathrm{CV}$ & $3,35 \pm 0,95$ & $3,45 \pm 1,22$ & $3,91 \pm 0,31$ & $4,02 \pm 1,99$ \\
\hline $\operatorname{Dureza}(\mathbf{N}) \pm C V$ & $80,00 \pm 0,00$ & $86,70 \pm 5,77$ & $71,70 \pm 4,03$ & $76,70 \pm 3,77$ \\
\hline Friabilidade & $0,29 \%$ & $0,08 \%$ & $0,16 \%$ & $0,50 \%$ \\
\hline
\end{tabular}

Legenda: $\mathrm{CV}=$ Coeficiente de variação. 
Tabela 3. Percentual de liberação de teofilina dos lotes A, B, C e D durante o ensaio de dissolução.

\begin{tabular}{cccccccc}
\hline \multirow{2}{*}{ Formulação } & \multicolumn{7}{c}{ \% fármaco liberado } \\
\cline { 2 - 9 } & $\mathbf{0 , 5 h}$ & $\mathbf{1 h}$ & $\mathbf{2 h}$ & $\mathbf{3 h}$ & $\mathbf{4 h}$ & $\mathbf{6 h}$ & $\mathbf{8 h}$ \\
\hline Lote A & 56,54 & 61,96 & 72,85 & 79,37 & 90,75 & 96,74 & 94,99 \\
Lote B & 25,37 & 30,74 & 37,02 & 43,94 & 48,85 & 53,26 & 58,19 \\
Lote C & 13,48 & 18,32 & 26,76 & 33,30 & 39,82 & 47,70 & 55,01 \\
Lote D & 10,02 & 12,57 & 19,64 & 23,10 & 28,45 & 29,72 & 43,66 \\
\hline
\end{tabular}

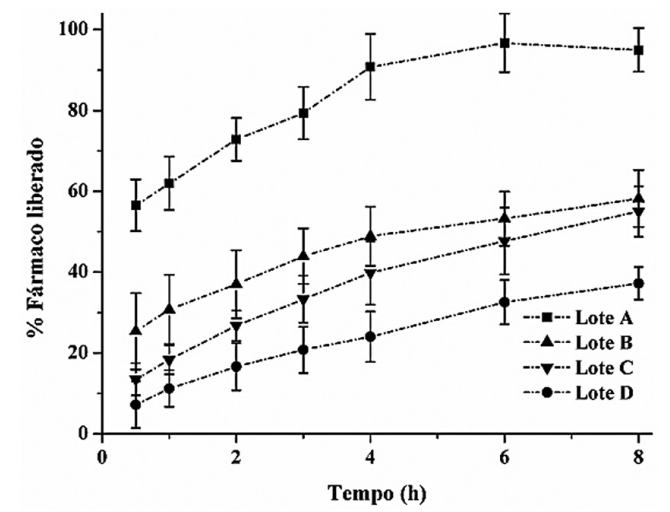

Figura 2. Gráfico dos perfis de dissolução dos lotes de comprimidos matriciais de teofilina durante 8 horas de ensaio.

apresentaram um grau de intumescimento praticamente constante durante o ensaio. Portanto, houve uma diminuição da taxa de erosão da matriz polimérica, refletindo em um maior controle da liberação da teofilina.

De acordo com o percentual de teofilina liberada em cada formulação ao longo do tempo (Tabela 3), observou-se que o lote $\mathrm{A}(\mathrm{GG})$ liberou muito rapidamente o fármaco, pois em apenas 30 minutos houve uma liberação maior que $50 \%$ de teofilina. O lote D (HPMC) foi o que apresentou maior retenção do fármaco, enquanto que os lotes $\mathrm{B}$ (GG:HPMC 3:1) e C (GG:HPMC 1:3) apresentaram boa capacidade no controle da liberação da teofilina.

A Goma guar constitui um material promissor frente à liberação controlada de fármacos em sistemas matriciais ${ }^{[19-21]}$. No entanto, este polissacarídeo utilizado sozinho não foi capaz de controlar a taxa de teofilina liberada, por isso foi associado a um polímero hidrofílico (HPMC), amplamente utilizado em comprimidos de liberação controlada ${ }^{[22-24]}$. Observou-se que a associação de 25\% de HPMC à matriz polimérica é suficiente para que ocorra um maior controle da liberação do fármaco.

A associação de Goma guar e HPMC em formulações de comprimidos matriciais demonstrou resultados bastante satisfatórios. A proporção entre esses polímeros é fator condicionante no processo de liberação do fármaco no sistema hidrofílico. Isso porque determina a formação da camada de gel ao redor da matriz e, consequentemente, a velocidade de dissolução, difusão do fármaco e erosão do sistema.

\section{Conclusões}

A utilização da Goma guar isolada, no desenvolvimento de comprimidos matriciais de liberação controlada, não apresentou resultados satisfatórios em relação ao controle da liberação da teofilina, mas quando associada à HPMC houve uma redução significativa do processo de erosão da matriz e, portanto, uma maior retenção do fármaco. Diante disso, comprimidos matriciais contendo Goma guar:HPMC, na razão 3:1, apresentaram a capacidade de controlar a liberação da teofilina, agregando valor à utilização de polímeros naturais no delineamento de formas farmacêuticas.

\section{Agradecimentos}

Agradecemos à Fundação Cearense de Apoio ao Desenvolvimento Científico e Tecnológico (FUNCAP) pelo auxílio financeiro e à Colorcon ${ }^{\circledR}$ (Brasil) pelo fornecimento do polímero hidroxipropilmetilcelulose $\left(\mathrm{METHOCEL}^{\mathrm{TM}}\right.$, HPMC K4M).

\section{Referências}

1. El-bagory, I., Barakat, N., Ibrahim, M. A., \& El-enazi, F. (2012). Formulation and in vitro evaluation of theophylline matrix tablets prepared by direct compression: effect of polymer blends. Saudi Pharmaceutical Journal, 20(3), 229-238. http:// dx.doi.org/10.1016/j.jsps.2011.11.007.

2. Freitas, A. B. R., \& Cavalcanti, O. A. (2004). Goma Guar como excipiente em matrizes hidrofílicas: avaliação do índice de hidratação. Acta Farmaceutica Bonaerense, 23(3), 334-338. Recuperado em 30 de abril de 2014, de http://www.latamjpharm. org/trabajos/23/3/LAJOP_23_3_1_10_I8YB5IGLFQ.pdf

3. Cavalcanti, O. A. (2002). Excipientes farmacêuticos: perspectivas dos polissacarídeos na pesquisa e desenvolvimento de novos sistemas de liberação. Arquivos de Ciências da Saúde Unipar, 6(1), 53-56. Recuperado em 30 de abril de 2014, de http:// revistas.unipar.br/saude/article/view/1151/1013

4. Pezzini, B. R., Silva, M. A. S., \& Ferraz, H. G. (2007). Formas farmacêuticas sólidas orais de liberação prolongada: sistemas monolíticos e multiparticulados. Revista Brasileira de Ciências Farmacêuticas, 43(4), 491-502. http://dx.doi.org/10.1590/ S1516-93322007000400002.

5. Lopes, C. M., Lobo, J. M. S., \& Costa, P. (2005). Formas farmacêuticas de liberação modificada: polímeros hidrofílicos. Revista Brasileira de Ciências Farmacêuticas, 41(2), 143-154. http://dx.doi.org/10.1590/S1516-93322005000200003.

6. Vueba, M. L., Batista de Carvalho, L. A. E., Veiga, F., Sousa, J. J., \& Pina, M. E. (2004). Influence of cellulose ether polymers on ketoprofen release from hydrophilic matrix tablets. European Journal of Pharmaceutics and Biopharmaceutics, 58(1), 51-59. http://dx.doi.org/10.1016/j.ejpb.2004.03.006. PMid:15207537.

7. Gonçalves-Araújo, T., Rajabi-Siahboomi,A. R., \& Caraballo, I. (2008). Application of percolation theory in the study of an extended release Verapamil hydrochloride formulation. International Journal of Pharmaceutics, 361(1-2), 112-117. http:// dx.doi.org/10.1016/j.ijpharm.2008.05.022. PMid:18621491.

8. Novak, S. D., Šporar, E., Baumgartner, S., \& Vrečer, F. (2012). Characterization of physicochemical properties of hydroxypropyl methylcellulose (HPMC) type 2208 and their influence on prolonged drug release from matrix tablets. Journal 
of Pharmaceutical and Biomedical Analysis, 66, 136-143. http:// dx.doi.org/10.1016/j.jpba.2012.03.032. PMid:22510313.

9. Barakat, N. S., Elbagory, I. M., \& Almurshedi, A. S. (2008). Controlled-release carbamazepine granules and tablets comprising lipophilic and hydrophilic matrix components. AAPS PharmSciTech, 9(4), 1054-1062. http://dx.doi.org/10.1208/ s12249-008-9140-y. PMid:18843537.

10. Srivastava, P., Malviya, R., Gupta, S., \& Sharma, P. K. (2010). Evaluation of various natural Gums as release modifiers in tablet formulations. Pharmacognosy Journal, 2(13), 525-529. http://dx.doi.org/10.1016/S0975-3575(10)80055-0.

11. Vendruscolo, C. W., Andreazza, I. F., Ganter, J. L. M. S., Ferrero, C., \& Bresolin, T. M. B. (2005). Xanthan and galactomannan (from M. scabrella) matrix tablets for oral controlled delivery of theophylline. International Journal of Pharmaceutics, 296(12), 1-11. http://dx.doi.org/10.1016/j.ijpharm.2005.02.007. PMid: 15885450 .

12. Wu, B., Chen, Z., Wei, X., Sun, N., Lu, Y., \& Wu, W. (2007). Biphasic release of indomethacin from HPMC/pectin/calcium matrix tablet: I. Characterization and mechanistic study. European Journal of Pharmaceutics and Biopharmaceutics, 67(3), 707-714. http://dx.doi.org/10.1016/j.ejpb.2007.04.016. PMid:17540549.

13. Hodges, L. A., Connolly, S. M., Band, J., O’Mahony, B., Ugurlu, T., Turkoglu, M., Wilson, C. G., \& Stevens, H. N. E. (2009). Scintigraphic evaluation of colon targeting pectinHPMC tablets in healthy volunteers. International Journal of Pharmaceutics, 370(1-2), 144-150. http://dx.doi.org/10.1016/j. ijpharm.2008.12.002. PMid:19114096.

14. Jian, H., Zhu, L., Zhang, W., Sun, D., \& Jiang, J. (2012). Galactomannan (from Gleditsia sinensis Lam.) and xanthan gum matrix tablets for controlled delivery of theophylline: In vitro drug release and swelling behavior. Carbohydrate Polymers, 87(3), 2176-2182. http://dx.doi.org/10.1016/j. carbpol.2011.10.043.

15. Serafin, W. E. (2005). Fármacos usados no tratamento da asma. In J. G. Hardman, L.E. Limbird, P. B. Molinoff, R. W. Ruddon, \& A. G. Gilman (Ed.), Goodman \& Gilman's: as bases farmacológicas da terapêutica (13. ed., pp. 409-412). Rio de Janeiro: Guanabara Koogan.
16. Agência Nacional de Vigilância Sanitária. (2010). Farmacopeia Brasileira. Brasília: Editora Fiocruz.

17. Efentakis, M., Vlachou, M., \& Choulis, N. H. (1997). Effects of excipients on swelling and drug release from compressed matrices. Drug Development and Industrial Pharmacy, 23(1), 107-112. http://dx.doi.org/10.3109/03639049709148487.

18. United States Pharmacopeial Convention .(2007). The United States Pharmacopeia (USP). Rockville: USP.

19. Gliko-Kabir, I., Yagen, B., Penhasi, A., \& Rubinstein, A. (2000). Phosphated crosslinked guar for colon-specific drug delivery: I. Preparation and physicochemical characterization. Journal of Controlled Release, 63(1-2), 121-127. http://dx.doi. org/10.1016/S0168-3659(99)00179-0. PMid:10640585.

20. Krishnaiah, Y. S. R., Karthikeyan, R. S., \& Satyanarayana, V. (2002). A three-layer guar gum matrix tablet for oral controlled delivery of highly soluble metoprolol tartrate. International Journal of Pharmaceutics, 241(2), 353-366. http://dx.doi. org/10.1016/S0378-5173(02)00273-9. PMid:12100863.

21. Singh, R., Maity, S., \& Sa, B. (2014). Effect of ionic crosslink on the release of metronidazole from partially carboxymethylated guar gum tablet. Carbohydrate Polymers, 106, 414-421. http:// dx.doi.org/10.1016/j.carbpol.2014.01.033. PMid:24721097.

22. Herder, J., Adolfsson, A., \& Larsson, A. (2006). Initial studies of water granulation of eight grades of hypromellose (HPMC). International Journal of Pharmaceutics, 313(1-2), 57-65. http:// dx.doi.org/10.1016/j.ijpharm.2006.01.024. PMid:16510256.

23. Ojoe, E., Miyauchi, E. M., Kaneko, T. M., Velasco, M. V. R., \& Consiglieri, V. O. (2007). Influence of cellulose polymers type on in vitro controlled release tablets containing theophylline. Revista Brasileira de Ciências Farmacêuticas, 43(4), 571-579. http://dx.doi.org/10.1590/S1516-93322007000400010.

24. Viridén, A., Abrahmsén-Alami, S., Wittgren, B., \& Larsson, A. (2011). Release of theophylline and carbamazepine from matrix tablets - consequences of HPMC chemical heterogeneity. European Journal of Pharmaceutics and Biopharmaceutics, 78(3), 470-471. http://dx.doi.org/10.1016/j.ejpb.2011.02.003. PMid:21316446.

Enviado: Abr. 30, 2014

Revisado: Ago. 21, 2014 Aceito: Fev. 26, 2015 